\title{
Transcriptional profile and serological studies of the European sea bass immune response against betanodavirus infections
}

\author{
P. Moreno , B. Lopez-Jimena , G. Scapigliati , F. Buonocore , E. Garcia-Rosado ${ }^{3}$, J.J. Borrego ${ }^{3}$, \\ J. Béjar , M.C. Alonso \\ ${ }^{1}$ Universidad de Málaga, Departamento de Microbiología, Facultad de Ciencias, 29071 Málaga, Spain \\ ${ }^{2}$ LifeArc Centre for Diagnostics Development. Nine, Edinburgh Bioquarter, 9 Little France Road, \\ Edinburgh, EH16 4UX, Scotland, United Kingdom \\ ${ }^{3}$ Department for Innovation in Biological, Agrofood and Forest Systems, University of Tuscia, Italy
}

\begin{abstract}
European sea bass (Dicentrarchus labrax) culture is highly affected by outbreaks of viral nervous necrosis disease, provoked by the nervous ne- crosis virus (NNV). This virus displays a single-stranded, positive-sense RNA genome, which is composed of two segments, RNA1, encoding the viral polymerase; and RNA2, encoding the capsid protein. Only two ge- notypes of NNV have been detected in sea bass to date, although showing very different levels of virulence. Specifically, RGNNV is highly virulent to sea bass, causing high mortality, whereas SJNNV replicates in sea bass brain without causing clinical signs. In the present work, the comparative analysis of the European sea bass immune response against isolates belonging to both viral genotypes has been performed. The immune response has been evaluated in brain and head kidney of experimentally infected sea bass by relative real-time PCR of genes involved in the type I interferon (IFN I) system (ifn-I, mxA, isg15, isg12), and genes related to inflammatory (il-8, tnf-a, il-10, tgf-b) and adaptive responses (tr-g, mhc-b). Ribosomal 18S RNA was used as reference endogenous gene. In addition, a serological study, consisting of the ELISA quantification of IgM in sera, was also performed. The transcription analyses of the innate defence-related genes point out the importance of this mechanism to control betanoda- virus infections. The results obtained showed a strong induction of ifn-I, mxA, isg 15 and isg 12 in both organs analysed, especially in response to the virus highly virulent to sea bass (RGNNV). However, the response was quicker in head kidney of SJNNVinoculated sea bass, suggesting that this genotype induces a more rapid systemic response. Regarding the inflam- matory response, RGNNV triggered a strong transcription of proin- flammatory genes in brain, which provides evidences about the importance of the inflammatory process in betanodavirus infection. Thus, the massive inflammatory process may be responsible for the eventual damage in nervous tissues, which would lead to fish dead. Finally, the high values of tr-g and mhc-b mRNA recorded in brain and the high IgM titer in sera, which was higher in SJNNV-inoculated fish, suggest that the adaptive response constitutes another important factor in the European sea bass immune response against betanodaviruses, both at systemic and at local level.
\end{abstract}

This study has been supported by the project AGL2017-84644-R (MINECO/AEI/FEDER, UE). P. Moreno was supported by a fellowship from Ministerio de Educación, Cultura y Deporte (FPU12/00265, Spanish Government).

\# Corresponding authors; E-mail address: mdalonso@uma.es (M.C. Alonso). 